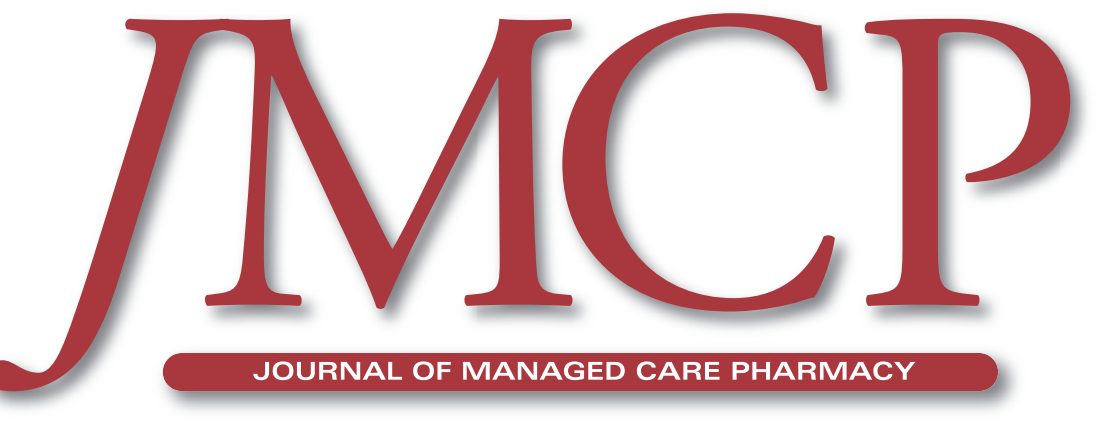

\title{
The AMCP Format for Formulary Submissions
}

\section{Version 3.0}

A Format for Submission of Clinical and Economic Evidence of

Pharmaceuticals in Support of Formulary Consideration

FMCP Format Executive Committee

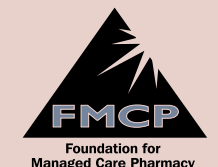

AMP

\section{Supplement}

January 2010

Vol. 16, No. 1-a

Continuing Education Activity 


\section{MCP}

Editor-in-Chief

Frederic R. Curtiss, PhD, RPh, CEBS

830.935.4319, fcurtiss@amcp.org

\section{Associate Editor}

Kathleen A. Fairman, MA

602.867.1343, kfairman@amcp.org

\section{Peer Review Administrator}

Jennifer A. Booker, 703.317.0725

jmcpreview@amcp.org

\section{Graphic Designer}

Margie C. Hunter

703.297.9319,mhunter@amcp.org

\section{Account Manager}

Bob Heiman, 856.673.4000

bob.rhmedia@comcast.net

\section{Publisher}

Judith A. Cahill, CEBS

Executive Director

Academy of Managed Care Pharmacy

This supplement to the Journal of Managed Care Pharmacy (ISSN 1083-4087) is a publication of the Academy of Managed Care Pharmacy, 100 North Pitt St., Suite 400, Alexandria, VA 22314; 703.683.8416; 703.683.8417 (fax).

Copyright $(\odot)$ 2010, Academy of Managed Care Pharmacy. All rights reserved. No part of this publication may be reproduced or transmitted in any form or by any means, electronic or mechanical, without written permission from the Academy of Managed Care Pharmacy.

POSTMASTER: Send address changes to JMCP, 100 North Pitt St., Suite 400, Alexandria, VA 22314.

\section{Supplement Policy Statement}

\section{Standards for Supplements to the} Journal of Managed Care Pharmacy

Supplements to the Journal of Managed Care Pharmacy are intended to support medical education and research in areas of clinical practice, health care quality improvement, or efficient administration and delivery of health benefits. The following standards are applied to all JMCP supplements to ensure quality and assist readers in evaluating potential bias and determining alternate explanations for findings and results.

1. Disclose the principal sources of funding in a manner that permits easy recognition by the reader.

2. Disclose the existence of all potential conflicts of interest among supplement contributors, including financial or personal bias.

3. Describe all drugs by generic name unless the use of the brand name is necessary to reduce the opportunity for confusion among readers.

4. Identify any off-label (unapproved) use by drug name and specific off-label indication.

5. Strive to report subjects of current interest to managed care pharmacists and other managed care professionals.

6. Seek and publish content that does not duplicate content in the Journal of Managed Care Pharmacy.

7. Subject all supplements to expert peer review.

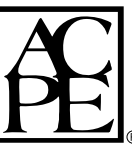

Pharmacists Accreditation and Credit Designation Statement

The Academy of Managed Care Pharmacy (AMCP) is accredited by the Accreditation Council for Pharmacy Education as a provider of continuing pharmacy education. AMCP designates this continuing education activity for 2.0 contact hours or 0.2 Continuing Education Units (CEUs). ACPE Universal Program Number: 0233-9999062-H01-P

For questions regarding the accreditation of this activity, please contact the AMCP Education and Meetings Department at 800.827.2626, ext. 604.

Release Date: January 1, 2010

Expiration Date: January 1, 2013

Type of Activity: Knowledge-Based

\section{Target Audience}

The target audience for this activity is pharmacists.

\section{Learning Objectives}

Upon completion of this program, the participant will be able to:

1. Identify the changes that were implemented in Version 3.0 of the AMCP Format for Formulary Submissions.

2. Describe the evidentiary requirements for models contained in Version 3.0 of the AMCP Format for Formulary Submissions

3. Specify the key general principles for unsolicited requests

4. Describe the role of the AMCP Format in evidence-based decision making

5. Explain the benefits of using cost-effectiveness models in assessing the value of health care technologies.

\section{Funding of This Learning Activity}

There is no commercial support of this learning activity.

\section{Fee Information}

There is no fee to participate in this learning activity.

\section{Authors}

FMCP Format Executive Committee (listed in Acknowledgements)

\section{Author Disclosures}

None of the members of the FMCP Format Executive Committee listed in the Acknowledgements received compensation for this update to the AMCP Format.
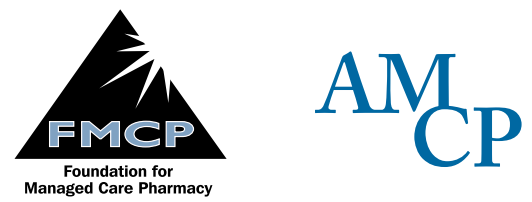

Academy of

Managed Care

Pharmacy ${ }^{\oplus}$

This supplement updates the June 2005 JMCP supplement for the AMCP Format Version 2.1, available at: $h t t p: / / w w w . a m c p . o r g / d a t a / j m c p / f o r m a t s u p p . p d f$ 


\title{
Table of Contents
}

\section{The AMCP Format for Formulary Submissions \\ Version 3.0}

\author{
FMCP Format Executive Committee
}

S3 Acknowledgements

S4 Preface

S4 - The Role of the AMCP Format

S5 - Confidentiality

S6 - Unsolicited Requests

S6 Requests for and Submission of Updates to Existing Dossiers

S6 - Multiple Dossiers Generally Are Not Needed

S6 - Implementation date for Version 3.0

S6 - Communication - The Key to Success

S7 Media for Dossier and Model Submissions

S7 - Conclusion

S8 Evidence Requirements for Formulary Submission

S8 1.0 Executive Summary - Clinical and Economic Value of the Product

S8 $\quad 1.1$ Clinical Benefits

S8 $\quad 1.2$ Economic Benefits

S8 $\quad 1.3$ Conclusions

S8 2.0 Product Information and Disease Description

S8 $\quad 2.1$ Product Description

S10 2.2 Place of the Product in Therapy

S10 2.2.1 Disease Description

S10 2.2.2 Approaches to Treatment

S11 2.2.3 Relevant Treatment Guidelines and Consensus Statements from National and/or International Bodies

S11 2.3 Evidence for Pharmacogenomic Tests and Drugs

S11 3.0 Supporting Clinical Evidence

S11 3.1 Summarizing Key Clinical Studies

S13 3.1.1 Published and Unpublished Data and Clinical Studies Supporting Labeled Indications

S13 3.1.2 Published and Unpublished Data and Clinical Studies Supporting Off-Label Indications

S13 3.1.3 Clinical Evidence Table Spreadsheets of all Published and Unpublished Studies

S14 3.1.4 Summary of Evidence from Secondary Sources 


\title{
Table of Contents
}

\author{
Continued
}

514

514

S14

S15

S16

S17

S17

S18

S19

S19

S19

S20

S20

521

521

S21

S22

S22

S22

S22

S22 Terms and Definitions

S26

References

S27 Appendices (available at www.fmcpnet.org)

A. Principles of a Sound Drug Formulary System

B. Sample Unsolicited Request Letter

C. Unsolicited Request Guidelines

D. Guidelines for Authors and Peer Reviewers of Economic Submissions to the British Medical Journal (M.F. Drummond)

E. Principles of Good Practice for Decision Analytic Modeling in Health-Care Evaluation: Report of the ISPOR Task Force on Good Research Practices Modeling Studies

F. Sample P\&T Committee Monograph

G. Formulary Leveraged Improved Prescribing: Evaluation Tool for Guiding Critical Formulary Decision-Making 
The Academy of Managed Care Pharmacy (AMCP) and the Foundation for Managed Care Pharmacy (FMCP) gratefully acknowledge the contributions of many individuals who have devoted substantial time, expertise and commitment in the preparation of the 3rd revision the AMCP Format for Formulary Submissions.

In particular, we are deeply indebted to the members of the FMCP Format Executive Committee for their continuing support of the missions of FMCP and AMCP and for their spirit of volunteerism that has ensured broad acceptance of the AMCP Format and completion of this version: Sean D. Sullivan, RPh, PhD, (Committee Chair), Professor of Pharmacy, Public Health and Medicine and Director, Pharmaceutical Outcomes Research and Policy Program (PORPP), University of Washington; Steven G. Avey, RPh, MS, Vice President, Managed Care, Partners Rx Management, LLC; Jennifer A. Flynn, Manager, US Outcomes Research, Eli Lilly and Company; Jeff Lee, PharmD, FCCP, Director, Applied Health Outcomes, Allergan, Inc.; Todd A. Lee, PharmD, PhD, Senior Investigator, Center for Management of Complex Chronic Care, Hines VA Hospital, Hines, IL; Bryan R. Luce, PhD, MBA, Senior Vice President, Science Policy, United BioSource Corp; Newell McElwee, PharmD, MSPH, Executive Director, US Outcomes Research, Merck \& Company, Inc.; Daniel Malone, RPh, PhD, Professor and Director, Division of Pharmaceutical Policy, University of Arizona College of Pharmacy; Peter J. Neumann, ScD, Director, Center for the Evaluation of Value \& Risk in Health, Professor, Tufts University School of Medicine Institute for Clinical Research and Health Policy Studies; Pete Penna, PharmD, President, Formulary Resources, LLC; Helen Sherman, PharmD, Clinical Pharmacist Consultant, Regence BlueCross BlueShield of Oregon; John Watkins, RPh, MPH, Pharmacy Manager, Formulary Development, Premera Blue Cross; and Richard N. Fry, RPh, FMCP Director of Programs and Committee staff liaison.

We are also grateful for the considerable contributions of the members of the Format Revision working groups: Jon Montague Clouse, BS Pharm, MS, Director, Pharmacoeconomic Evaluations, UnitedHealth Pharmaceutical Solutions; Lida R. Etemad, PharmD, MS, Senior Director, PDL Development, UnitedHealth Pharmaceutical Solutions; Helen Lee Phan, PharmD, MBA, Clinical Pharmacist, CareFirst BlueCross BlueShield; Iris Tam, PharmD, Director, Institutional Medical Communications, Genentech; David L. Veenstra, PharmD, PhD, Associate Professor, Pharmaceutical Outcomes Research and Policy Program and Institute for Public Health Genetics, University of Washington; T. Jeffrey White, PharmD, MS, Director, Clinical Analytic Strategies, WellPoint; and Richard J. Willke, PhD, Head, Global Health Economics and Outcomes Research, Primary Care, Pfizer Inc.

\section{DISCLOSURES}

This project was sponsored by the Academy of Managed Care Pharmacy and received no external funding or commercial support. 
PREFACE

\section{THE ROLE OF THE AMCP FORMAT}

The evidence requirements contained herein are intended for use by manufacturers of pharmaceuticals, biologics and vaccines who are responding to an unsolicited request from a healthcare system to support reimbursement and/ or formulary placement consideration of a new product, new indication, or new formulation of an existing product.

The Format and other formulary submission templates and guidelines support the informed selection of pharmaceuticals, biologics and vaccines by:

a. Standardizing and communicating product and supporting program information requirements

b. Requiring projections of product impact on both the organization and its enrolled patient population

c. Requesting information on the value of products

d. Making evidence and rationale supporting all choice(s) more clear, transparent and evaluable by decision makers

The AMCP Format's process is designed to maintain a high standard of objectivity to achieve two important goals. First, it is intended to improve the timeliness, scope, quality and relevance of information available to a healthcare system's evaluators and ultimately to its Pharmacy and Therapeutics (P\&T) or other technology assessment committees. Further, by assessing the healthcare system impact of using a product, the evidence requested can improve the healthcare system's ability to compare the effects of formulary alternatives on clinical outcomes, value, and economic consequences for the entire healthcare system. According to Neumann, the type of rigorous clinical and economic analysis called for in the Format "forces and focuses discussions about the value of health and medical services within a clear theoretical framework. It generates a more careful consideration of available evidence and sheds light on how to target resources to particular clinical practices or subgroups of patients." ${ }^{1}$ However, it is important that this information is weighed in the context of other values such as equity, social justice, and the health of individuals as opposed to populations, the "rule of rescue" and other elements of rational resource allocation. ${ }^{2,3,4}$

Second, the AMCP Format streamlines the evidence acquisition and review process for healthcare system staff. By clearly specifying the standards of evidence implicit in the existing formulary process, the Format furnishes pharmaceutical manufacturers with consistent direction concerning the nature and format of information that is expected. In addition, the standardized format allows healthcare system staff to formally evaluate the completeness of submissions received and to easily add the results of the healthcare system's own systematic literature reviews and analysis. Manufacturers should understand that submission of information in the recommended format does not guarantee approval of their product for formulary listing. Manufacturers and healthcare systems should view discussion about, and subsequent submission of a dossier, as a process to improve the quality and layout of information 
PREFACE (CONTINUED) provided, but not as a formula for approval. The Format offers a clear, shared vision of the requirements to facilitate the collaboration necessary between healthcare systems and manufacturers to support appropriate and evidencebased drug product evaluation. Recognizing that manufacturers may not have all the requested evidence, especially for new products, the Format describes the information requirements necessary to support a comprehensive assessment of the proposed product.

The Academy of Managed Care Pharmacy continues to view the AMCP Format as a template or guide, and not a mandate. As such, it does not claim to establish a standard of practice for managed care pharmacy that is directly applicable to the evidence needs of each healthcare system. It is up to individual healthcare systems to decide if and how they will use the AMCP Format and how they will operate their formulary review processes.

While the AMCP Format does not specify methods for assessing clinical benefit, harms or economic impact, the evidence presented should meet accepted standards of evidence-based medicine and health technology assessment. It is the manufacturer's responsibility to utilize appropriate study designs, analytic techniques and data sources, and the requester's responsibility to critically evaluate the evidence supplied.

\section{CONFIDENTIALITY}

The confidentiality of evidence dossiers. The confidentiality of evidence dossiers has been an area of concern since AMCP published the first version of the Format in October, 2000. Manufacturers have expressed concern that confidential information submitted as part of an evidence dossier-e.g., unpublished studies, off-label information, economic modeling data-will become publicly available, thus exposing sensitive data to competitors, and potentially alarming regulatory authorities worried about misleading promotion. To a large extent, the concerns should be addressed through compliance with FDA guidance on unsolicited requests and with appropriate confidentiality agreements between the healthcare system and the manufacturer. Healthcare systems should be aware that the ability of manufacturers to provide complete information is dependant on the recipient to preserve the confidentiality of that information. We note that evidence dossiers submitted to government authorities in the US, the UK and certain other countries are made available to the public but commercial-in-confidence information, when properly identified by the manufacturer, is redacted for the online version of the report. Special arrangements with public payers, which require public disclosure of information received, may be necessary.

Warning: Product dossiers prepared in accordance with the evidence requirements contained in the AMCP Format for Formulary Submissions may contain off-label information and information deemed proprietary by the product manufacturer. Therefore, such dossiers may only be distributed in response to an unsolicited request.

Manufacturers may require requesting organizations to sign a confidentiality agreement before providing a dossier. Such agreement may also be required where prepublication data are shared with a healthcare system. Healthcare systems should be willing to sign such agreements and adhere to their terms.

AMCP recommends that manufacturers place a statement on the dossier that it is being provided in response to an unsolicited request. 
PREFACE

(CONTINUED)

\section{UNSOLICITED REQUESTS}

To qualify as an unsolicited request, the request for information must be truly unsolicited. Specifically, the inquiry must be initiated by the requester (formulated in his/her own mind) without prompting, suggestion or solicitation by the drug manufacturer or its employees. The general principles and well-accepted standards that have been established in this field, include: 1) the manufacturer does not prompt or encourage requests, 2) responses focus on data rather than company-generated discussions of those data, 3) individuals with the appropriate scientific and medical training prepare responses, 4) responses do not deliberately go beyond the scope of the request, 5) responses do not include promotional materials, and 6) responses are objective, balanced and scientifically rigorous.

Therefore, at no time, shall an evidence dossier in the AMCP Format be sent to a healthcare system without an authentic, validated unsolicited request from the healthcare system directly to the manufacturer. Any violation of this rule, intentional or not, jeopardizes the regulatory safe harbor for unsolicited requests that allows industry to prepare and respond to requests for product dossiers in the AMCP Format, as well as the Academy's original intent and mission for the AMCP Format. ${ }^{5}$ See Appendix $C$ for a more detailed treatment of this issue.

\section{REOUESTS FOR AND SUBMISSION OF UPDATES TO EXISTING DOSSIERS}

During recent discussions, FDA regulatory staff has stated that individual unsolicited requests are required to obtain updates to product dossiers that include information on unlabeled uses. To avoid health systems having to submit potentially numerous unsolicited requests FDA staff agreed that health systems may include in their original unsolicited request for an evidence dossier, a statement requesting any new published and unpublished information on labeled and unlabeled uses, including updated dossiers for the specific product. NOTE: The request for updated information must pertain only to the specific information or dossier included in the original unsolicited request. Also, the request for updates must specify a length of time, e.g., 6 months.

\section{MULTIPLE DOSSIERS GENERALLY ARE NOT NEEDED}

AMCP recognizes that the evidence requirements contained herein may not meet the evidence needs of all healthcare systems. For example, WellPoint ${ }^{6,7}$ and The Regence Group ${ }^{8,9}$ have recently issued their own detailed evidence requirements. AMCP has learned from WellPoint and The Regence Group that unless otherwise specified by the healthcare system, manufacturers should submit a single evidence dossier prepared according to the AMCP Format with an addendum that contains the additional evidence requirements requested by the healthcare system.

\section{IMPLEMENTATION DATE FOR VERSION 3.0}

Dossiers under development may be converted to Version 3.0 with relative ease. New dossiers developed after January 1, 2010 should be completed using Version 3.0.

\section{COMMUNICATION - THE KEY TO SUCCESS}

AMCP believes that to conduct an appropriate technology assessment function for the purpose of making coverage and formulary decisions, there should be substantial on-going communication between the healthcare 
PREFACE (CONTINUED) system and the pharmaceutical company throughout the assessment process in order to manage expectations and maximize the quality of available evidence. Those organizations that have been early adopters of the AMCP Format have expressed the importance of and concern for good communication. When a dossier is requested from a healthcare system, it is important for that organization to communicate to the pharmaceutical company basic information such as time-lines, the evaluation process, potential data sources, any special needs that might exist, etc. This also gives the pharmaceutical company an opportunity to discuss its available evidence. If they cannot submit specific studies or provide a certain component of the economic analysis, it is better to understand the limitations up front. AMCP does not suggest that healthcare systems should significantly alter or disrupt their normal lines of communication with pharmaceutical manufacturers. However, both parties should recognize that when there is a high level of collaboration, there is a relative increase in the chances that the process will be smoother and the quality of the dossiers submitted will be higher. ${ }^{10}$

\section{MEDIA FOR DOSSIER AND MODEL SUBMISSIONS}

$\mathrm{AMCP}$ and FMCP encourage manufacturers to submit dossiers in an electronic format rather than in print. This will help reduce resource expenditures and improve healthcare system staff's ability to transfer evidence directly into P\&T committee submission monographs. As with printed dossiers, manufacturers and healthcare systems must ensure that all tenets of the FDA's unsolicited request process are followed (see Appendix C). In addition manufacturers must provide a transparent, unlocked copy of the model without a graphical interface. It should be presented electronically as an Excel workbook, ASCII tab-delimited file or an alternative electronic format that is agreed upon by the requesting organization or its consultants and the manufacturer.

\section{CONCLUSION}

The persistent rise in health care expenditures, particularly with medical technology, is attributable in part to the unwillingness of the American public to accept limits on the use of health care services. This is also a major factor contributing to resistance to the use of cost-effectiveness analysis in the United States as a policy making tool. ${ }^{1}$ Other contributing factors such as a lack of understanding about the conceptual approach, a mistrust of methods and motives, and regulatory and legal barriers may be more easily overcome. However, Daniels and Sabin, writing in Health Affairs in 1998 stated, "To change that culture requires a concerted effort at education, and education requires openness about the rationales for managed care plan's decisions." ${ }^{11}$ By adhering to careful and thoughtful decision-making processes that provide the rationales for limits, health care systems will be able to show, over time, that "arguably fair decisions are being made and that those making them have established a procedure we should view as legitimate."11 AMCP and FMCP believe that the AMCP Format is a valuable tool that will continue to help healthcare systems establish a record of commitment to rational evidencebased decision-making, thus gaining the confidence of patients, clinicians, payers and members. 
EVIDENCE

REQUIREMENTS

FOR FORMULARY

SUBMISSION

\subsection{EXECUTIVE SUMMARY - CLINICAL AND ECONOMIC VALUE OF THE PRODUCT}

Based on the clinical and economic evidence presented in Sections 2 through 5 of the dossier, justify the value of the product. This section of the submission requirements replaces Section 4 of the previous version (Version 2.1) of the AMCP Format for Formulary Submissions and represents the principal opportunity for a manufacturer to briefly summarize the value of its product. The manufacturer should briefly describe the clinical and economic information presented in the dossier using the layout prescribed in Sections 1.1 and 1.2 and state the expected per unit product cost. Based on this information, the manufacturer should articulate a value argument to justify these expected expenditures for this product in the context of its anticipated effects on the clinical evidence, health outcomes, and the economic consequences for the healthcare system.

1.1 CLINICAL BENEFITS: Begin with the FDA-approved indication for the drug and a short (1 to 2 paragraph) synopsis of the efficacy and safety information (from the prescribing information and clinical trials). Summarize (no more than 1 page) the clinical benefits of the PROPOSED THERAPY, in terms of:

- Efficacy and Effectiveness

- Safety/tolerability

- Shortcomings of current treatment and the unmet medical need that the PROPOSED THERAPY addresses

1.2 ECONOMIC BENEFITS: Summarize (no more than 1 page) the economic benefits of the PROPOSED THERAPY, in terms of:

- Cost per unit

- Context of the proposed cost: potential clinical benefits provided (including quality of life benefits) and potential economic benefits (including savings or cost offsets)

- Shortcomings of other therapies

Briefly present results of any observational research or economic data, with inclusion of the PMPM or ICER result at minimum. Briefly summarize other published information on the cost or economic impact of the product (such as impact of resource utilization or other cost offsets).

1.3 CONCLUSIONS. (no more than $1 / 2$ page) Summarize the value of the PROPOSED THERAPY in 1 to 2 paragraphs. Highlight key points regarding the clinical and economic advantages and uniqueness of the product are highlighted. Finally, based on the information presented in Sections 2 to 5 that follow, the conclusions should include a statement regarding the expected impact of the product, relative to other available treatment options both pharmaceutical and non-pharmaceutical.

\subsection{PRODUCT INFORMATION and DISEASE DESCRIPTION}

2.1 PRODUCT DESCRIPTION. (20 pages maximum) Manufacturers are required to provide detailed information about their product. They should compare the new product with other agents commonly used to treat the condition, whether or not these products are currently on the healthcare system's formulary.

The product description consists of information that traditionally has been incorporated in a product monograph, Package Insert (PI) or formulary kit as described below. It also contains information that goes beyond the scope of the package insert, monograph and formulary kit that can only be provided pursuant to an unsolicited request. 
EVIDENCE

REQUIREMENTS

FOR FORMULARY

SUBMISSION
NOTE: Basic product information should be provided, including a brief discussion of what the product is, and any significant attributes that define the product's place in therapy (e.g. kinetics, adverse event profile, etc.). Verbatim language from the Package Insert (PI) should not be supplied here. If there is not substantive data and information that can be provided beyond what is in the PI, these sections should be left blank and the reader referred to the copy of the PI which is in the Appendix. In those cases where one or more of these attributes (pharmacology, pharmacokinetics, pharmacodynamics, contraindications, warnings, precautions, adverse events, interactions, and/or dosing) is of major significance in defining the value of a drug, additional information beyond that found in the PI should be provided.

The following are the components that should be supplied:

a. Generic, brand name and therapeutic class of the product

b. All dosage forms, including strengths and package sizes

c. The National Drug Code (NDC) for all formulations

d. The ASP and WAC cost per unit size. (The payers contract price, if available, should be included as well)

e. AHFS or other Drug Classification

f. FDA approved indication(s) and the date approval was granted (or is expected to be granted). Also other significant off-label uses and potential new indications being studied

g. Pharmacology*

h. Pharmacokinetics/Pharmacodynamics*

i. Contraindications*Warnings/Precautions/Adverse Effects*

j. Interactions* with suggestions on how to avoid them

- Drug/Drug

- Drug/Food

- Drug/Disease

k. Dosing and Administration*

1. Access, e.g. restrictions on distribution, supply limitations, anticipated shortages, and/or prescribing restrictions

m. Co-Prescribed / Concomitant Therapies, including dosages, and recommended use of other agents or treatments with the product

n. Concise comparison of PI information with the primary comparator products in the same therapeutic area to include: dosing, indications, pharmacokinetic/ pharmacologic profile, adverse effects, warnings, contraindications, interactions and other relevant characteristics. (Expand as appropriate for the therapeutic class.) The material may include a discussion of comparator product(s) or services that the proposed product is expected to substitute for, or replace. This information should be presented in tabular form. If direct head-to-head trials have been conducted on the drug and its comparators, this should be noted here, and the reader referred to the review of those trials in Section 3 of the dossier.

*Verbatim language from the Approved Package Insert should not be supplied here. If there is not substantive data or information that can be provided beyond the label, these sections should be left blank and the reader referred to the copy of the PI which is in the Appendix. 
EVIDENCE

REQUIREMENTS

FOR FORMULARY

SUBMISSION
2.2 PLACE OF THE PRODUCT IN THERAPY (NOTE: FOR PRODUCTS WITH MULTIPLE INDICATIONS, THE FOLLOWING INFORMATION SHOULD BE PROVIDED FOR EACH INDICATION.) IDEALLY, INFORMATION SHOULD BE PROVIDED IN TABLE OR BULLET FORMAT. INFORMATION PRESENTED IN THIS SECTION SHOULD BE BRIEF. DO NOT DUPLICATE INFORMATION PRESENTED IN SECTIONS 3.0, 4.0, AND 5.0.

\section{THIS SECTION INCLUDES THREE PARTS:}

2.2.1 DISEASE DESCRIPTION. (limit to $1-2$ pages per disease) The intent is to give the reader a good overall sense of the disease. The disease description should be brief, and should include the disease and characteristics of the patients who are treated for the condition. Manufacturers should provide a description of specific patient subpopulations in which the drug is expected to be most effective, if known. Include clinical markers, diagnostic or genetic criteria, or other markers, if known, that can be used to identify these subpopulations. Present a brief summary of information from the literature for each topic. Ideally, information should be provided in table or bullet format.

Disease specific descriptive information should include, but not be limited to:

a. Epidemiology and relevant risk factors, with a focus on identifiable subpopulations that would be appropriate for the use of the product

b. Pathophysiology

c. Clinical presentation

d. Societal, humanistic and/or economic burden

2.2.2 APPROACHES TO TREATMENT. (limit to 1-2 pages per major indication) The key questions to address are: How is the disease/condition currently treated? How does the new product fit into standard or existing therapy? Provide a VERY brief summary of information from the literature for each topic; do not duplicate information included in other sections:

a. Approaches to treatment-principal options/practice patterns

b. A description of alternative treatment options (both drug and nondrug)

c. The place and anticipated uses of the proposed therapy in treatment (e.g. first line), especially for any subpopulations that can be targeted for the use of the product

d. Proposed ancillary disease or care management intervention strategies to be provided by the manufacturer that are intended to accompany the product at launch

e. The expected outcomes of therapy, e.g. a cure, palliation, relief of symptoms, etc. Describe any clinical markers that that are linked to disease outcome, e.g. LDL lowering

f. Description of other drug development or post-marketing obligations as required by the FDA such as a Risk Evaluation and Mitigation Strategy (REMS), Phase IV trial, patient registry, restricted distribution channel, and other elements designed to assure the safe use of the product

g. Other key assumptions and their rationale 
EVIDENCE

REQUIREMENTS

FOR FORMULARY

SUBMISSION
2.2.3 RELEVANT TREATMENT GUIDELINES AND CONSENSUS STATEMENTS FROM NATIONAL AND/OR INTERNATIONAL BODIES. This section should describe the treatment guideline's position on the therapy. Include position statements and validated tools from national organizations and international HTA bodies, e.g., NICE. Next, an attempt should be made to generalize these findings to the populations of the requesting organization. Discuss the implications of any differences that exist between the literature and typical practice patterns and patient populations. When more than one disease is addressed, complete the description for each separate condition. The requesting organization and the manufacturer should determine the relevant treatment options for comparison during the initial pre-submission meeting.

2.3 EVIDENCE FOR PHARMACOGENOMIC TESTS AND DRUGS. In considering the appropriate use of genetic testing to guide drug therapy (variously referred to as 'pharmacogenomics', 'pharmacogenetics', 'personalized medicine', or 'targeted therapy'), clinicians and healthcare system decision makers must consider the accuracy with which a test identifies a patient's genetic status (analytic validity), clinical status (clinical validity), and the risks and benefits resulting from test use (clinical utility). ${ }^{12}$ The following evidence should be presented as appropriate in support of submissions involving pharmacogenomic testing, or drugs for which pharmacogenomic testing is available:

Analytic Validity

- Accuracy with which a particular genetic characteristic can be identified using a genetic test in relation to professional standards and federal regulation requirements

Clinical Validity

- Strength of the association between the genetic variant(s) and clinical outcome(s) (e.g., efficacy, adverse drug reaction)

- Expected prevalence of genetic variant(s) in target population; positive predictive value (PPV) and negative predictive value (NPV) of test

Clinical Utility

- Effectiveness and safety of the clinical intervention implemented as a result of the genetic test, as per Sections 3.1-3.3 of the Format; consider inclusion of quantitative risk-benefit decision analytic modeling (Section 4.1.1 of the Format)

\section{Cost Effectiveness}

- Expected difference in costs and outcomes with pharmacogenomic testing compared to usual care, as per Section 4.2.3 of the Format, including cost offsets from changes in drug utilization and health outcomes

\subsection{SUPPORTING CLINICAL EVIDENCE}

3.1 SUMMARIZING KEY CLINICAL STUDIES: (2 page maximum per study) Please complete evidence tables in the format requested by the healthcare system or as suggested in appendix F. Submit summaries of all relevant clinical studies that have been conducted, whether published or not, in each of the categories listed in Sections 3.1.1 and 3.1.2. Refer also to the Relevance Criteria below. 
EVIDENCE

REQUIREMENTS

FOR FORMULARY

SUBMISSION
NOTE: To avoid duplication, include ALL relevant clinical studies (summaries and evidence tables) only in Section 3.0 and all relevant economic studies (summaries and evidence tables) only in Section 5.0. However, if a study reported both clinical and economic outcomes, include the study summary in this section. Tabulate the clinical results in Section 3.1.1. To avoid duplication, Section 5.0 should refer to the summary description in Section 3.1. Economic outcomes should be tabulated in Section 5.0.

Include detailed summaries for all relevant clinical studies.

All of the following items that apply should be included in the study summaries:

a. Name of the clinical trial or study and publication citation(s)

b. Objective, location and study date

c. Trial design, randomization and blinding procedures

d. Setting, inclusion and exclusion criteria

e. Sample characteristics (demographics, number studied, disease severity, co-morbidities)

f. Drop-out rates and procedures for handling drop-outs (ITT, per protocol, etc.)

g. Treatment: dosage regimens, washout period, etc.

h. Clinical outcome(s) measures

- Outcomes evaluated

- Delineate primary vs. secondary study endpoints and their corresponding results

i. Other outcome measures (e.g., patient-reported outcomes)

- Principal findings

j. Statistical significance of outcomes and power calculations

k. Validation of outcomes instruments (if applicable)

1. Generalizability of the population treated

m. Study limitations, as stated by the authors

n. Publication citation(s)/references used including funding source of the study

RELEVANCE CRITERIA-Use the following criteria to determine relevance:

a. Relevant studies that provide clinical information that may impact formulary decisions, including but not limited to:

- Safety, including total number of patients exposed to the drug

- Efficacy, effectiveness and comparative effectiveness

- Identification of patient subgroups, practice settings, etc. in which use of the drug may be more appropriate

b. Include all Phase 3 clinical trials

c. In general, include all large, randomized controlled trials

d. Include smaller studies, e.g., Phase 2 trials, only if they contain relevant information that is not provided by larger studies

e. Include studies conducted in settings outside the U.S. if they add new information not contained in the U.S. trials

f. Do not include basic pharmacologic studies, e.g., Phase 1 studies

g. Do not include purely pharmacokinetic studies, unless the value proposition is based on the pharmacokinetic properties of the product, or the studies identify an appropriate patient subgroup 
EVIDENCE

REQUIREMENTS

FOR FORMULARY

SUBMISSION
3.1.1 INCLUDE ALL RELEVANT PUBLISHED AND UNPUBLISHED CLINICAL STUDIES SUPPORTING LABELED INDICATIONS:

1. Placebo-controlled safety and efficacy trials

2. Prospective effectiveness and comparative effectiveness trials, including pragmatic trials

3. Open-label safety extension studies

4. Prospective studies examining other non-economic endpoints such as health status measures and patient-reported outcomes. If the instruments utilized in these studies are supported by previous validation and reliability studies, also reference these studies

5. Unpublished data: Provide as much detail as can be disclosed

3.1.2 INCLUDE ALL PUBLISHED AND UNPUBLISHED DATA AND CLINICAL STUDIES SUPPORTING OFF-LABEL INDICATIONS:

1. Include all relevant studies of the types listed in 3.1.1 above

2. Include off-label indications that are reasonably likely to be considered by practitioners, based on the available supporting evidence. Provide contact information for questions about other uses

3. Unpublished data: Provide as much detail as can be disclosed

4. This constitutes an unsolicited request for ALL relevant studies supporting off-label uses of the product

Studies reported in this section should be summarized in a clear, concise format and include all relevant positive and negative findings. (see above relevance criteria) If the results of a trial have been published as more than one paper, all may be combined into one summary and one evidence table row, citing all the articles from which data have been drawn and clearly stating the total number of subjects. The payer is particularly interested in head-to-head comparison clinical studies between the proposed product and the principal comparators. Summaries of trial results of key comparator products are desirable but not required. Discuss important study findings and comment on their implications for different patient populations. Systematic reviews or meta-analyses are to be included in Section 5.0

Include citations for all known published clinical studies in the bibliography section. Reprints of relevant published studies should be available upon request. Where possible, provide a link to original sources if they are free.

Additional items:

- Include relevant data and findings from the Center for Drug Evaluation and Research's Office of Drug Safety

- Include confirmation that trials for the product are registered in a public trials registry and provide access information (e.g. www. clinicaltrials.gov)

- Include list of ongoing clinical trials and links to their registry information

3.1.3 CLINICAL EVIDENCE SPREADSHEETS OF ALL PUBLISHED AND UNPUBLISHED TRIALS. Information from all relevant studies on the product should be summarized in evidence tables in the format requested by the healthcare system or as suggested in Appendix F, Template for P\&T Committee Monograph. Include negative or null findings as well as positive findings. 
EVIDENCE

REQUIREMENTS

FOR FORMULARY

SUBMISSION
When including pragmatic trials or observational studies that contain both clinical and economic endpoints include the clinical endpoints in the clinical evidence table and include the economic endpoints in the table that summarizes published CEAs (Section 5.1.2).

Evidence tables should include the following data elements:

- Citation, (if unpublished, give abstract information or indicate "data on file")

- Treatments

- Sample size and length of follow-up

- Inclusion/exclusion criteria

- Design

- Primary Endpoints

- Secondary Endpoints

- Results: Provide an explicit statement of effect size, not just relative risk reduction and/or statistical significance. Within the Results column, include a table of key results

- Statistical significance

3.1.4 SUMMARY OF EVIDENCE FROM SECONDARY SOURCES. Relevant evidence may be available from a variety of secondary sources. The following may be submitted. Summaries should be concise, focusing only on the major conclusions:

- Cochrane Collaboration systematic reviews

- Formal, published systematic reviews from peer-reviewed journals

- Agency for Healthcare Research and Quality (AHRQ) evidence summaries

- Health technology assessments from other recognized agencies, public or private, including reviews from other countries

- Evidence-based clinical practice guidelines, medical society position statements, etc. These documents should include explicit evidence grading criteria

- Compendia officially recognized by the Secretary of Health and Human Services that list the drug. If these references are available only by subscription, provide PDF documents or reprints of the relevant content

Summaries of information from one of these sources should be limited to a maximum of one page.

4.0 ECONOMIC VALUE and MODELING REPORT. (maximum 20 pages) "Far better an approximate answer to the right question, which is often vague, than an exact answer to the wrong question, which can always be made precise." John W. Tukey, 1962

4.1 MODELING OVERVIEW. This section presents an overview of the rationale, approach, and suggested methods for developing a decision-analytic based cost-effectiveness model. The intent of the model is to quantify for the healthcare system the risk-benefit tradeoff of the product, and its economic value.

4.1.1 UTILITY OF MODELING FOR DECISION-MAKING. Available data on the clinical benefits and harms and economic impact of the drug under consideration are provided in Sections 3 and 5 of the AMCP Format, and are the core of evidence-based decision-making. These data, however, may have important limitations for decision-making. For example, 


\section{EVIDENCE REQUIREMENTS FOR FORMULARY SUBMISSION}

- Randomized controlled trials (RCTs) may not include all relevant comparator interventions

- The duration of follow-up in RCTs may be limited

- Patient populations in RCTs may not be reflective of plan populations

- Safety data may be limited, or from disparate sources

- Healthcare cost impacts may not be generalizable across payers

These limitations have led to recent efforts in comparative effectiveness research to improve the quantity and quality of information available to healthcare decision makers. Comparative effectiveness data - derived from studies including relevant populations, comparators, and outcomes-will prove highly valuable to healthcare system formulary decision makers, and should be reported in Section 3 of the Format. These data are more likely (and should be expected) to be available for more mature products. In addition, evidence may be generated through pay for performance or coverage with evidence development schemes. Synthesis and evaluation of these data will remain challenging, however, and are unlikely to be available for new products.

Decision-analytic based, cost-effectiveness models are one of the best available means to assess the overall potential value of healthcare technologies. They are disease-based and take into account the impact of the new technology on the clinical outcomes for the target population. Typically, they include evidence on the incidence of the disease or condition in the target population, the medical care required to diagnose and treat the disease, the relative and absolute risk reductions offered by the technology, survival and quality of life impacts, and the costs of the interventions. Decision models can provide:

- An explicit framework for decision-making

- Synthesis of evidence on health consequences and costs from many different sources

- Formal assessment of uncertainty

- A quantitative measure of clinical risk-benefit

- Explicit and evaluable assumptions

- Specificity for a product's role or place in therapy

- Benchmarks against which the product's future performance can be measured

Models are not without challenges. In particular, because of the complexity and inherent required assumptions, models can be perceived a 'black-box' approach or biased. The AMCP Format has been developed to help address these limitations by providing a consistent format for conducting and reporting cost-effectiveness models to improve their transparency and acceptability.

\subsubsection{TYPES OF MODELS Cost-effectiveness models}

There are several types of models that can be helpful for managed care decision makers. The focus of the AMCP Format is the clinical and economic value of drug therapies for plans and their members. Evaluations that include impacts on patients - e.g., morbidity and mortality - and on healthcare costs are thus most relevant, and termed in general 'cost-effectiveness models.' These models are primarily useful for assessing the overall clinical risk-benefit and economic value of a drug in relation to drugs in 
EVIDENCE

REQUIREMENTS

FOR FORMULARY

SUBMISSION its class and other healthcare interventions in general, and are the primary focus of this Section. There are several specific types of cost-effectiveness models, which are discussed in the Methods section below.

\section{Budget impact models}

Budget impact models are not intended to establish the overall value of healthcare technologies because they do not include the full impact of the technology on clinical and patient outcomes. They can be useful for estimating system-wide (e.g., pharmacy and medical) budget impacts, however, and are commonly used by managed care payers. These models, as defined here, estimate drug costs, healthcare cost offsets, and adverse event costs, as well as the expected utilization in the healthcare system, to derive projected per member per month costs. Budget impact models utilize clinical data and can be relatively complex, and thus should follow the recommendations in this section, as well as published best practices ${ }^{13}$ (see also Appendix E).

\section{Financial models}

Financial models provide an estimate of the financial impact of a new technology on the pharmacy budget only because they typically include drug costs, network or other discounts, rebates, co-payment and other benefit design impacts, but no evaluation of clinical effects or other economic consequences. Payers usually have the necessary internal resources to develop such models. Although these models may be useful for negotiations between manufacturers and payers, they are not central to the evidence- and value-based decision making process, and are not addressed further in the Format.

\subsubsection{OTHER CONSIDERATIONS}

- When a product is intended for treatment of more than one disease or indication, its impact should be modeled for each, unless a reasonable case can be made for a single model, such as may be the case for budget impact models.

- Models that have been previously developed may be adapted for use according to the AMCP Format. An existing model should be modified to follow the general framework described in this document and must be able to demonstrate the system-wide impact of introducing the product to healthcare system formularies. Evidence supporting the validity of existing models should be provided, as well as sufficient documentation on their design, functioning, and data inputs.

- Cost-effectiveness analyses conducted alongside RCTs, particularly when of sufficient size and follow-up, can provide useful and sometimes substantial evidence of economic value. Cost-effectiveness models should be considered complementary to such studies, allowing for the adjustment of healthcare resource use, unit costs, effectiveness, and practice patterns.

- Lastly, users of this document should recognize the Format is a set of recommendations on the types of evidence and reporting formats that are likely to be useful for managed care decision makers. We recognize the need for flexibility, however. Specific requirements are determined by individual managed care organizations, and may consist of data requests or methods beyond those outlined in this document. 


\section{EVIDENCE REQUIREMENTS FOR FORMULARY SUBMISSION}

\subsection{MODELING APPROACHES AND METHODS 4.2.1 APPROACH AND FRAMEWORK Guidelines}

Manufacturers should consult with healthcare system staff in the early stages of model development to identify optimal modeling approaches and ensure the incorporation of appropriate comparator products and endpoints. In general, the cost-effectiveness framework should consider recommendations published by the Panel on Cost-Effectiveness in Health and Medicine convened by the U.S. Public Health Service, ${ }^{14}$ and the model should follow the guidance provided by the International Society for Pharmacoeconomics and Outcomes Research (ISPOR) Good Practice Modeling Principles. ${ }^{15}$ (see also Appendix E)

The model should be disease-based, and depict the following:

a. Disease or condition, patient population, natural history, clinical course and outcomes

b. Primary treatment options and the treatment process for each option - preferably based on treatment guidelines or actual practice

c. Costs of product and other medical resources consumed within each clinical pathway

d. Outcomes of therapy for each clinical pathway

e. Incremental cost and outcomes analysis presented in cost/consequences tables and as cost-effectiveness ratios

\section{Analytic framework}

The general category of 'cost-effectiveness' models includes analyses that value outcomes by assessing clinical events, life expectancy, and/or quality-adjusted life-years (QALYs). Clinical events are more readily interpretable by clinicians and allow for direct assessment of the impact of clinical data, but cost per event avoided calculations are not comparable across disease areas. In contrast, QALYs allow for assessment of overall healthcare value, but may be more difficult to interpret from a healthcare system perspective. It is thus recommended that clinical events, life expectancy, and QALYs all be assessed-with the latter two outcomes primarily relevant for lifetime timeframe analyses. The results should be reported separately, as outlined subsequently in this section. Exclusion of any of these endpoints should be justified.

\section{Modeling technique}

There are several decision-analytic based approaches to constructing disease-based cost-effectiveness models, primarily: 1) decision trees, 2) Markov (cohort) models, and 3) patient-level simulation (discrete event simulation). There are advantages and disadvantages to each technique, primarily related to the conflicting factors of transparency and data availability vs. the complexity of many diseases and their treatments. It is recommended that the simplest feasible modeling approach be utilized - in other words, the model should be sophisticated enough to capture the key aspects of the disease and treatments, yet be well supported by highquality data that are available to and interpretable by the user.

\section{Perspective and Timeframe}

The payer perspective is recommended for the primary analysis, with optional perspectives (i.e., societal, employer) conducted as secondary evaluations. The model should consider a time horizon that is appropriate 
EVIDENCE

REQUIREMENTS

FOR FORMULARY

SUBMISSION to the disease being studied and reflect the decision-making and financial and budget constraints of the healthcare system. Multiple timeframes are recommended for chronic disease-e.g., 5-year, 10-year, and lifetime. Adjustment for time preference should be incorporated as appropriate and follow US PHS Panel recommendations (i.e., discounting both future costs and health effects at a 3\% annual rate). ${ }^{14}$

4.2.2 DATA SOURCES. The identification, selection, interpretation, and use of data to inform the model is the most important aspect of the modeling process, and should receive ample attention from model developers and users. The analysis should be based on the highest-quality and most up to date clinical, epidemiologic, patient, and economic data available. The process for identifying, evaluating, and selecting all of the data in the model should be clear and systematic.

It is extremely important that modeled claims for cost-effectiveness derive from data from one or more comparative effectiveness trials. These should:

- Directly compare and quantify treatment effects and other relevant patient-reported outcomes (including quality of life)

- Assess patient and community preferences for alternative therapies

- Quantify costs and benefits over the natural course of the disease

- Assess resources used to support alternative therapies

- Evaluate the impact of uncertainty on the claims made for alternative therapies

Parameter estimates used in the model for the drug under consideration should be closely linked with the evidence provided in all Sections of the Format. All necessary assumptions should be clearly stated. In addition to the identification of base-case estimates for the model, ranges for parameters should be determined and well-referenced.

\section{Drug effectiveness}

Randomized controlled trial data should form the basis of all efficacy or effectiveness estimates, and justification should be provided for inclusion and exclusion of any RCTs potentially relevant to the analysis.

\section{Drug safety data}

Clinically relevant adverse events observed in RCTs should be included in the model, as well as safety signals derived from appropriate observational studies. A wide range of estimates should be explored given the challenge of accurately ascertaining the likelihood of low-probability events.

\section{Economic data}

Unit costs data ideally would be based on healthcare system data. If specific healthcare system data are not available, costs from representative U.S. private payers, or Medicare, can be used. Decision-analytic models should be sufficiently flexible to adapt the input assumptions to conform to local practice patterns.

\section{Utilities}

Preference estimates should be derived from studies surveying either patients or the general population, using a direct elicitation method or an instrument such as the EQ-5D or HUI.

\section{Demographic and practice pattern data}

Demographic and practice pattern data from the healthcare system should be incorporated as appropriate to improve the relevance of the model. 
EVIDENCE

REQUIREMENTS

FOR FORMULARY

SUBMISSION

\section{Surrogate markers}

When surrogate markers are used to model longer-term outcomes, specific evidence should be provided supporting their validity.

\section{Expert opinion}

Data derived from expert panels are not generally acceptable, especially for key effectiveness or safety variables. However, this approach may be reasonable for other variables where estimates are not available through literature, databases, trials or other normal sources. In such cases, the expert assumptions should be clearly stated and thoroughly tested in sensitivity analyses. Inputs obtained from an expert panel should be modifiable in case local opinion leaders disagree with the panel members.

\section{Efficacy vs. effectiveness}

When feasible and scientifically plausible, efficacy results from RCTs should be transformed into effectiveness parameters. This may involve inclusion of an adherence parameter into the model based on observational data. Documentation and clear description of the methodology will be necessary in order for healthcare system staff to evaluate the validity of this approach.

\subsubsection{ANALYSIS}

\section{Base-case estimates}

The expected (average) clinical and economic outcomes should be calculated for each strategy evaluated, as well as incremental costs, effectiveness, and cost effectiveness. Differences in the absolute risk of events should be determined, and healthcare cost offsets vs. drug costs independently determined. Clinical risk-benefit tradeoffs should be explicitly presented and discussed.

\section{Sensitivity analysis}

Comprehensive one-way sensitivity analysis of all parameters in the model is strongly recommended, including assessment of impacts on both incremental effectiveness (e.g., QALYs) and cost-effectiveness. Scenario analyses testing the assumptions used in the model are also highly recommended. The 3-5 parameters and 2-3 assumptions that have the greatest impact on the results should be identified. Probabilistic sensitivity analysis and the generation of cost-effectiveness scatter plots and acceptability curves are recommended, particularly for more complex models.

\subsection{MODELING REPORT AND INTERACTIVE MODEL}

4.3.1 TRANSPARENCY. Transparency and clarity of presentation are a necessity. The need for and value of transparency is widely recognized and can provide some protection against the negative effects of bias and error. The users of models need to be able to understand all steps in the modeling process to improve their understanding of the key factors and variables in the model and its limitations. ${ }^{16}$ Therefore, researchers are encouraged to focus efforts on the clarity and transparency of results. Detailed descriptions that explain the flow of data through the model are recommended. All calculations should be explained in a simple straightforward manner to allow a non-health economist to comprehend the analysis. This information and references should be accessible both in the report format as well as shown directly in the model to maximize the ease of review.

Listed below are the recommended requirements for modeling reports and interactive models. 
EVIDENCE

REQUIREMENTS

FOR FORMULARY

SUBMISSION
4.3.2 MODELING REPORT FORMAT. The modeling report should follow the format: 1) Introduction/Background, 2) Methods, 3) Results, 4) Limitations, 5) Discussion. A 500 word abstract following this same format should be provided on the first page of the modeling report, and include an explicit description of the key drivers of the model results identified in sensitivity and scenario analyses.

Below are the minimum recommended figures and tables. Multiple tables in each category (e.g. Table la, lb, etc.) may be used if needed.

Figure 1. Provide a figure displaying the structure of the model (e.g., a decision tree or Markov model). A simplified schematic diagram may be used for ease of presentation, but a detailed figure should also be included.

Table 1. Provide a table listing all of the model inputs, including probabilities, costs, and utility estimates if appropriate. Provide a range of values upon which sensitivity analyses are based for each input.

a. Include references in the table for all inputs, including ranges

b. Note in the table estimates that lack supporting evidence

Table 2. Provide an explicit list of model assumptions, including assumptions about comparator interventions, clinical events, patient management, and costs.

Table 3. Present the disaggregated results in a table (e.g., cost-consequence style, with costs presented separately from health outcomes). Data presented in this format are more easily understood and interpreted by healthcare system formulary committees. The following specific data should be presented for each strategy:

a. The projected clinical events (e.g., heart attacks, cirrhosis, recurrence)

b. The life expectancy and QALY estimates

c. Total healthcare costs

d. The cost of implementing therapy and the resulting cost offsets

e. Incremental cost-effectiveness ratios

Figure 2. Present one-way sensitivity analyses on all model inputs in a figure (e.g., tornado diagram) or a table.

a. Clearly present the model inputs or assumptions that drive the difference in 1) costs, 2) effects, and 3) incremental cost-effectiveness

b. When appropriate, present multi-way (e.g., 2-way, best/worst case scenario, probabilistic) sensitivity analyses

\subsubsection{INTERACTIVE MODEL}

Model characteristics

To improve transparency and ease of use, it is recommended that models be implemented in spreadsheet software. Other software packages should only be used if the user a) is familiar with them, and b) agrees with the manufacturer to their use. Custom software models are generally discouraged, but may be feasible for use if clearly documented in peer-reviewed publications and a users manual. Interactive models should have the following characteristics:

- All data and calculations relevant to the cost-effectiveness model should be contained in the spreadsheet and visible to the user 
EVIDENCE

REQUIREMENTS

FOR FORMULARY

SUBMISSION
- All inputs should be modifiable by the user

- To the extent feasible, the model, its logic and its calculations should be clear and self-documenting, using best practices for formatting, comments, and explanatory guides such as text boxes

- Allow for analysis of relevant sub-populations (age, gender, comorbidities) where applicable

- Allow the healthcare system to incorporate its own data (membership size, prevalence rates, cost estimates, etc.) in place of default data, such as national norms

- Provide automated 1-way sensitivity analysis

\section{Model accessibility}

It is recommended that the healthcare system require that an interactive model be made available electronically, preferably after meeting with the manufacturer to discuss its design, results, and use. If the manufacturer will not provide an interactive model for the payer's use, a clear statement to this effect and standing policy should be provided in the modeling report. Alternative approaches include interactive modification of the model with a representative of the manufacturer, although such arrangements are significantly less desirable.

Model users should recognize that input parameters must be plausible, and many combinations of inputs in complex models will not be selfconsistent. Thus, users should modify model inputs based on available data and reasonable assumptions.

\subsection{OTHER SUPPORTING EVIDENCE}

5.1 SUMMARIZING OTHER RELEVANT EVIDENCE: (2 page maximum per study) Provide summaries of other relevant supporting evidence including, but not limited to, retrospective studies that provide information not available from clinical trials, meta-analyses and systematic reviews of clinical, quality of life and economic outcomes, comparative observational studies of effectiveness and harms, assessments of adherence or persistence, studies of patient preference, predictive risk models, and indirect comparisons of clinical benefit using Bayesian or other appropriate methods, whether published or not. Conduct and reporting of studies of the type described above should follow accepted practice as evidenced by published methodology and reporting guidelines from reputable professional societies or government agencies.

5.1.1 INCLUDE PUBLISHED AND UNPUBLISHED STUDIES SUPPORTING LABELED AND OFF-LABEL INDICATIONS. Refer to Section 3.1 and 3.1.1 for items to be included in the study summaries and for relevance and grading criteria. In addition, summaries of relevant economic studies should include the following:

- Definition of economic endpoints (mean overall cost, cancer-related cost, \$/LYG, \$/QALY, etc.) including references for standard of care costs

- Data sources for economic endpoints

- Statistical methods/math used to calculate endpoints

- Modeling methodology (if applicable)

- Sensitivity analysis (if applicable)

- Unpublished data: Provide as much detail as can be disclosed

Include complete citations for all studies summarized in this 


\section{TERMS AND DEFINITIONS}

section. Reprints of relevant published studies should be available upon request. Where possible, provide a link to original sources if they are free of charge.

5.1.2 EVIDENCE TABLE SPREADSHEETS. Information from all studies described in this section should be summarized in evidence tables (spreadsheet format). Include negative or null findings as well as positive findings. Use a standard evidence table format, such as that contained in Appendix F, Template for P\&T Monograph. Refer to Section 3.1.3 for suggested data elements.

\subsection{SUPPORTING INFORMATION}

6.1 REFERENCES CONTAINED IN DOSSIERS. Include citations for all known published clinical and economic studies in the bibliography section. Reprints of relevant published studies should be available upon request. Include the PI in the Appendix and where possible, provide a link to original sources if they are free.

6.2 DOSSIERS AND ECONOMIC MODELS. Media: AMCP and FMCP encourage manufacturers to submit dossiers in an electronic format rather than in print. This will help reduce resource expenditures and improve healthcare system staff's ability to transfer evidence directly into P\&T committee submission monographs. As with printed dossiers, manufacturers and healthcare systems must ensure that all tenets of the FDA's unsolicited request process are followed (see also Appendix C). In addition manufacturers must provide a transparent, unlocked copy of the model without a graphical interface. It should be presented electronically as an Excel workbook, ASCII tab-delimited file or an alternative electronic format that is agreed upon by the requesting organization or its consultants and the manufacturer.

Transparency: The model should be transparent, i.e., designed to allow staff or consultants to investigate the assumptions and calculations, and to perform independent sensitivity analyses by varying individual parameters. The requesting organization will retain this model for internal analyses and will not release it to any other party. Manuscripts that support the development and reporting of the model should be either attached as appendices or made readily available upon request.

\section{Terms and Definitions}

Budget Impact Models: See definition in Section 4.1.2.

Care Pathways: A general method of using predetermined, time-staged, evidence-based actions for managing the care of patients who have clearly defined diagnoses or require certain procedures. Ideally, care pathways should be applicable to the management of patients moving among a managed health care system's multiple levels of care and practice settings. Other terms for care pathways include clinical care plans, clinical pathways, critical pathways, care guides, and care maps. ${ }^{17}$

Cost-Benefit Analysis: An analytical technique derived from economic theory that enumerates and compares the net costs of a health care intervention with the benefits that arise as a consequence of applying that intervention. For this technique, both the net costs and the benefits of the health intervention are expressed in monetary units. ${ }^{17}$

Cost-consequence Analysis: An analytical technique that compares the 


\section{TERMS AND DEFINITIONS}

health intervention of interest to one or more relevant alternatives, listing the cost components and various outcomes of each intervention separately. This type of economic analysis does not indicate the relative importance of the components listed and leaves it to the decision maker to form his or her own view. ${ }^{17}$

Cost-Effectiveness Analysis: A systematic method of comparing two or more alternative programs by measuring the costs and consequences of each. A distinguishing feature of cost-effectiveness analysis is that the consequences (health outcomes) of all programs to be compared must be measured in the same common units-natural units related to the clinical objective of the programs (e.g., symptom-free days gained, cases prevented, quality of life years gained) ${ }^{17}$

Cost-Minimization Analysis: A type of pharmacoeconomic analysis comparing two alternative therapies only in terms of costs because their outcomes (effectiveness and safety) are found to be or expected to be identical. ${ }^{17}$

Cost-Utility Analysis: A specific type of cost-effectiveness analysis that compares two or more alternative choices in terms of both their costs and outcomes, where the outcomes are measured of utility or preference, often as a quality-adjusted life years gained. Cost-utility analysis can be considered the "gold standard" methodology for evaluating the cost-effectiveness of health care choices. ${ }^{17}$

Cost-Effectiveness Model: These models are used to establish the value of a new technology relative to an appropriate comparator, and often use decision analysis techniques. They can be based directly on clinical trials. They are disease-based and account for the impact of new technology on clinical outcomes (efficacy, adverse events), resource use and costs in the short and long-term. They also reveal the relation between data inputs and assumptions and outcomes. These models can be used to conduct a cost-benefit, costminimization, cost-effectiveness, and/or cost-utility analysis. ${ }^{17}$

Decision Analysis: A quantitative approach to decision making under uncertainty in which all relevant elements of the decision - alternative actions, chance events (along with their probabilities of occurrence), and final consequences - are stated explicitly in a model. Multiple types of data can be incorporated from a variety of sources. This model typically takes the form of a decision tree or an influence diagram and permits the decision maker to determine systematically the relative value of alternative courses of action. ${ }^{17}$

Decision Tree: A schematic diagram depicting the logical structure of a choice under conditions of uncertainty, including all relevant alternative decisions available to the decision maker as well as the values and probabilities of all relevant downstream consequences. ${ }^{17}$

Dossier: A detailed report (in paper and electronic form) for each product submitted by the manufacturer for consideration that contains 1) clinical and economic data from published and unpublished studies and 2) a diseasebased economic model to project the potential impact that introducing the product would have on health and economic consequences occurring across the entire system.

Effectiveness: The actual effects of treatment by the drug under "real life" conditions [patients not always remembering to take their doses, physicians often not prescribing the lowest FDA recommended doses, side effects not all controlled, etc]. "Head to head" effectiveness studies with similar medications are preferable. 


\section{TERMS AND DEFINITIONS}

Efficacy: The potential effects of treatment by the drug under optimal circumstances [e.g. patients all taking their doses at the right times, physicians prescribing FDA recommended doses, side effects appropriately monitored, etc]. Efficacy studies are typically the foundation of new drug submissions to the FDA. Studies that compare the efficacy of similar drugs, rather than just efficacy compared to placebo are preferable.

Evidence-Based Medicine (EBM): An approach to health care decision making in which the decision maker is aware of all the relevant evidence and its strengths and weaknesses and is then able to apply that knowledge to decisions. EBM, therefore, consists of clinical expertise and patient preferences combined with critical appraisal of clinical research, with the goal of providing optimal individual patient care. Optimal care thus takes into account patient outcomes and the relative efficiencies among competing alternatives, as demonstrated in the medical literature. This approach to patient care demands that the decision makers' expertise and the appraisal of the clinical evidence base are current and up to date. ${ }^{17}$

Evidence-Based Medicine-Alternate Definition: The conscientious, explicit, and judicious use of current best evidence in making decisions about the care of individual patients. The practice of evidence based medicine means integrating individual clinical expertise with the best available external clinical evidence from systematic research.

Formulary: A periodically updated list of medications, related products and information, representing the clinical judgment of physicians, pharmacists, and other experts in the diagnosis and/or treatment of disease and promotion of health.

Formulary System: An ongoing process whereby a health care system, through its physicians, pharmacists and other health care professionals, establishes policies on the use of drugs, related products and therapies, and identifies drugs, related products and therapies that are the most medically appropriate and cost-effective to best serve the health interests of the patient populations of the healthcare systems it represents.

Health Economics: A discipline that analyses the economic aspects of health and health care and that usually focuses on the costs (inputs) and the consequences (outputs) of health care interventions using methods and theories from economics and medicine. ${ }^{17}$

Health-Related Quality of Life (HRQOL): A broad theoretical construct developed to explain and organize measures concerned with the evaluation of health status, attitudes, values and perceived levels of satisfaction and general well being with respect to either specific health conditions or life as a whole form the individual's perspective. (see Patient-Reported Outcomes). ${ }^{17}$

Markov Model: A complex health economics treatment model that describes the natural history of particular diseases, with or without treatment. To capture all critical events, Markov models can categorize health status with a higher level of detail and divide the model's time perspective into finer intervals than is possible with decision trees. ${ }^{17}$

Model: In the context of health care evaluation, a model is an analytic methodology that accounts for events over time and across populations, that is based on data drawn from primary and/or secondary sources and whose purpose is to estimate the effects of an intervention on valued health consequences and costs. ${ }^{15}$ 


\section{TERMS AND DEFINITIONS}

Modeling: The development of a simplified representation of a system (e.g. population). A particular model may be analytical, visual or both. In pharmacoeconomics specifically or health economics in general, analytical models can be used to pose and answer questions about interventions that cannot be directly answered by clinical trials due to time and financial constraints. ${ }^{17}$

Outcomes Research: The scientific discipline that evaluates the effect of health care interventions on patient-related, if not patient-specific, clinical, humanistic and economic outcomes. Outcomes research is generally based on the conceptual framework that evaluation of treatment alternatives involves the simultaneous assessment of multiple types of outcomes that are diseaserelated. ${ }^{17}$

Patient-Reported Outcomes: An umbrella term that includes outcome data reported directly by the patient. It is one source of data that may be used to describe a patient's condition and response to treatment. It includes such outcomes as global impressions, functional status, well-being, symptoms, healthrelated quality of life, satisfaction with treatment and treatment adherence. ${ }^{13}$

Pharmacoeconomics: The scientific discipline that assesses the overall value of pharmaceutical health care products, services and programs. Of necessity, it addresses the clinical, humanistic and economic aspects of health care interventions in the prevention, diagnosis, treatment and management of disease. Pharmacoeconomics thus provides information critical to the optimal allocation of heath care resources. The field encompasses experts in health economics, risk analysis, technology assessment, clinical evaluation, epidemiology, decision analysis and health services research. ${ }^{17}$

Quality-Adjusted Life Year (QALY): A universal health outcome measure applicable to all individuals and all diseases, thereby enabling comparisons across diseases and across programs. A QALY combines, in a single measure, gains or losses in both quantity of life (mortality) and quality of life (morbidity). ${ }^{17}$

Rule of Rescue: A term applied to the ethical imperative to save individual lives regardless of the cost if rescue measures are available. Regarding the distribution of health care services, the "rule of rescue" supplements rather than substitutes for the evidence-based consideration of comparative cost-effectiveness. For example, Australia's Pharmaceutical Benefits Advisory Committee considers the rule of rescue a relevant factor when the cost-effectiveness level is unacceptable and:

1. No alternate pharmacological or non-pharmacological intervention exists to treat patients with the identified condition

2. The defined condition must be severe, progressive and expected to lead to premature death

3. The defined condition must apply to only a very small number of patients ${ }^{4}$

Sensitivity Analysis: A way to analyze the impact of uncertainty in an economic analysis or a decision (see Decision Analysis, Modeling, CostEffectiveness Model). The simplest form of sensitivity analysis is a one-way analysis where the value of one variable is changed while keeping the other variables constant, and the impact on results evaluated. ${ }^{17}$

Tornado Diagram: A set of one-way sensitivity analyses displayed in a single graph, with the most critical variable in terms of impact at the top of the graph and the rest ranked according to their impact thereafter; hence the "tornado" 
REFERENCES Accessed December 10, 2009. December 10, 2009. 11, 2009. 1998;17(5):50-64. impact analysis. Value Health. 2007;10(5):336-47. Medicine. New York, NY: Oxford University Press; 1996. transparent, be reasonable. Value Health. 2003;6(1):6-8. Pharmacoeconomics and Outcomes Research. 2003. 2009. Manag Care. 2004;10(5):308-12. Available at: http://www.ajmc.com/media/pdf/ AJMC04MayNeumann308_12.pdf. Accessed December 10, 2009.

2. Gibson JL, Martin DK, Singer PA. Priority setting for new technologies in medicine: a transdisciplinary study. BMC Health Serv Res. 2002;2(1):14. Available at: http://www. biomedcentral.com/1472-6963/2/14. Accessed December 10, 2009.

3. Daniels N. Four unsolved rationing problems. Hastings Cent Rep. 1994;24(4):27-29.

4. Richardson J, McKie J. The rule of rescue. Working paper 112. Centre for Health Program Evaluation, Health Economics Unit, Monash University, West Heidelberg, Australia. Available at: http://www.buseco.monash.edu.au/centres/che/pubs/wpl12.pdf.

5. Salter FJ, Kramer PF, Palmer-Shevlin NL. Pharmaceutical industry medical communications departments: regulatory and legal perils and pitfalls. Drug Info J. 2000;34:1009-15.

6. WellPointNextRx. The WellPoint Outcomes Based Formulary. WellPoint health technology assessment guidelines: drug submission guidelines for new products, new indications, and new formulations. September 2008. Available at: https://www.wellpointnextrx.com/ shared/noapplication/f1/s0/t0/pw_ad080613.pdf. Accessed December 10, 2009.

7. WellPointNextRx. The WellPoint Outcomes Based Formulary. WellPoint health technology assessment guidelines: drug submission guidelines for re-evaluation of products, indications, and formulations. September 2008. Available at: https://www.wellpointnextrx. com/shared/noapplication/f1/s0/t0/pw_ad080614.pdf. Accessed December 10, 2009.

8. RegenceRx. AMCP dossier request form. 2009. Available at: http://www.regencerx.com/ docs/amcp-dossier-request-form.pdf. Accessed December 10, 2009.

9. The Regence Group. Medication policy development and review process. September 7 , 2007. Available at: http://blue.regence.com/policy/medication/introduction.html. Accessed

10. Foundation for Managed Care Pharmacy. Response to comments for AMCP's format for formulary submissions Version 2.0. Alexandria, VA. October 2002. Available at: http:// www.fmcpnet.org/cfr/waSys/f.cfc?method=getListFile\&id=A2D3AAB3. Accessed December

11. Daniels N, Sabin J. The ethics of accountability in managed care reform. Health Affairs.

12. U.S. Department of Health and Human Services, Food and Drug Administration, Center for Drug Evaluation and Research, Center for Biologics Evaluation and Research and Center for Devices and Radiologic Health. Draft guidance for industry: pharmacogenomic data submissions. November 2003. Available at: http://www.fda.gov/OHRMS/ DOCKETS/98fr/2003d-0497-gdl0001.pdf. Accessed December 10, 2009.

13. Mauskopf JA, Sullivan SD, Annemans L, et al. Principles of good practice for budget impact analysis: Report of the ISPOR Task Force on good research practices-budget

14. Gold MR, Siegel JE, Russell LB, Weinstein MC, eds. Cost-effectiveness in Health and

15. Weinstein MC, O’Brien B, Hornberger J, et al. Principles of good practice for decision analytic modeling in health-care evaluation: report of the ISPOR Task Force on good research practices--modeling studies. Value Health. 2003;6(1):9-17.

16. Garrison LP. The ISPOR Good Practice Modeling Principles-a sensible approach: be

17. Berger ML, Bingefors, K, Hedblom ED, Psashos CL, Torrance GW, Smith MD, eds Health Care Cost, Quality, and Outcomes; ISPOR Book of Terms. International Society for

18. Sackett DL, Rosenberg WM, Gray JA, Haynes RB, Richardson WS. Evidence based medicine: what it is and what it isn't. BMJ. 1996;312(7023):71-2. Available at: http://www. bmj.com/cgi/content/full/312/7023/71?view=long\&pmid=8555924. Accessed December 10 , 
APPENDICES (hyperlinked)
To access these appendices to the AMCP Format, Version 3.0, go to www.fmcpnet.org.

- Appendix A: Principles of a Sound Drug Formulary System

- Appendix B: Sample Unsolicited Request Letter

- Appendix C: Unsolicited Request Requirements

- Appendix D: Guidelines for Authors and Peer Reviewers of Economic Submissions to the British Medical Journal (M.F. Drummond)

- Appendix E: Principles of Good Practice for Decision Analytic Modeling in Health-Care Evaluation: Report of the ISPOR Task Force on Good Research Practices-Modeling Studies

- Appendix F: Sample P\&T Committee Monograph

- Appendix G: Formulary Leveraged Improved Prescribing: Evaluation Tool for Guiding Critical Formulary Decision Making 


\section{The AMCP Format for Formulary Submissions, Version 3.0}

Continuing education credit for this activity is processed through AMCP at www.amcp.org (CE/CME Center) only. No mailed forms will be accepted.

The posttest worksheet is provided to assist you in marking your answers prior to entering the online CE center for submission; these pages cannot be submitted for CE credits.

In order to receive $\mathrm{CE}$ credit for this activity, you must complete the following forms online:

1. The posttest for this program, "The AMCP Format for Formulary Submissions, Version 3.0" is accessible at the AMCP.org CE/CME Center. You must receive a score of $70 \%$. You will have 2 opportunities to pass the posttest.

2. Activity Evaluation Form.

Upon successful completion of this activity, you will automatically receive your CE, statement as proof of completion. Your CE credits will be archived and tracked for you on the AMCP.org CE/CME Center site. All information is kept confidential.

\section{Posttest Worksheet: The AMCP Format for Formulary Submissions, Version 3.0}

1. If no substantive data and information are available beyond what is contained in the product Package Insert (PI), Section 2.1 (Product Description) should contain the verbatim language from the PI.

a. True

b. False

2. The AMCP Format supports the informed selection of pharmaceuticals, biologics and vaccines through which of the following processes?

a. Standardizing and communicating product and supporting program information requirements

b. Requiring projections of product impact on both the organization and its enrolled patient population

c. Requesting information on the value of products

d. Making evidence and rationale supporting all choice(s) more clear, transparent and evaluable by decision makers

e. All of the above

3. The Executive Summary (Section 1.0) should contain all of the following information EXCEPT:

a. Summary of the clinical benefits of the proposed therapy in terms of safety, efficacy and effectiveness

b. Cost per unit

c. A summary of the key drivers of the model results

d. Summaries of the clinical and economic shortcomings of current therapies

4. Which of the following statements is TRUE?

a. AMCP Format-based dossiers may not meet the evidence requirements of all health care systems.

b. WellPoint and The Regence Group have issued their own evidence requirements.

c. Dossiers submitted to WellPoint and The Regence Group may be prepared according to the AMCP Format.

d. Dossiers prepared according to the AMCP Format and submitted to WellPoint and The Regence Group must include an addendum that contains their additional evidence requirements.

e. All of the above

5. For products with multiple indications, manufacturers are to prepare a dossier for each indication.

a. True

b. False
6. Communication has been identified as a key element for success when using the AMCP Format process. All of the following are TRUE as they relate to evaluating medications for formulary inclusion, EXCEPT:

a. There should be substantial on-going communication between the health care system and the pharmaceutical company.

b. Health care systems and pharmaceutical companies should discuss in advance any special evidence requirements.

c. If a pharmaceutical company communicates that they will have a dossier developed by launch but it will not have every piece of information, the health system should tell the company a review will not immediately take place.

d. Knowing what a company will and will not be able to supply is helpful in advance.

e. All of the previous statements are TRUE.

7. In terms of FDA guidelines and the AMCP Format, which of the following statements is TRUE?

a. Individual unsolicited requests are required to obtain product dossier updates.

b. Submission of an authenticated unsolicited request must always precede submission of an evidence dossier in the AMCP Format.

c. Health care systems may include in their initial unsolicited request a statement requesting dossier and product evidence updates for a specific period of time.

d. The request for updated information must pertain to the dossier included in the original unsolicited request.

e. All of the above

8. In addition to a description of the product, Section 2.0 should contain all of the following EXCEPT:

a. Detailed summaries of direct head-to-head trials conducted between the product and its comparators

b. Description of specific patient subpopulations targeted by the product

c. Pathophysiology and clinical presentation of the disease

d. Description of how the product fits into standard or existing therapy

e. Treatment guidelines and position statements from international health technology assessment entities 
9. Which of the following statements about economic models is FALSE?

a. Cost-effectiveness models are most valuable when they are transparent.

b. Health plans should be able to insert their own data into a model to make it more relevant to their environment.

c. The AMCP Format specifies the exact methods that must be used in an economic evaluation.

d. Cost-effectiveness models should assess clinical events, life expectancy, and quality-adjusted life-years (QALYs).

10. General principles and well-accepted standards for unsolicited requests include which of the following?

a. The manufacturer may not prompt or encourage requests.

b. Responses must focus on data rather than company-generated discussions of those data.

c. Individuals with the appropriate scientific and medical training must prepare responses.

d. Responses must not deliberately go beyond the scope of the request.

e. All of the above

11. Effectiveness is defined as the potential effects of treatment by a drug under optimal circumstances. Effectiveness studies are typically the foundation of new drug submissions to the FDA.

a. True

b. False

12. Regarding the submission of models in AMCP Format-based dossiers, all of the following statements are true EXCEPT:

a. Manufacturers are strongly urged to provide cost-effectiveness models.

b. Budget impact models can also be used to determine the overall value of new technologies.

c. One of the goals of cost-effectiveness models is to synthesize evidence on health consequences and costs from many different sources.

d. Cost-effectiveness models assist in creating health system performance benchmarks.

e. Financial models are not central to the evidence- and valuebased decision making process.

13. Regarding model characteristics, which of the following statements is FALSE?

a. Dossiers should include a transparent, unlocked model spreadsheet that allows users to make changes in multiple elements.

b. While models implemented in spreadsheet software are recommended, custom software models with a graphical interface are also highly desirable.

c. All data and calculations relevant to the model should be visible to the user.

d. The model should provide automated l-way sensitivity analysis.

14. Concerns regarding the confidentiality of the contents of dossiers should be addressed through compliance with FDA rules on unsolicited requests and with appropriate confidentiality agreements between health care systems and the manufacturer. This statement is:

a. True

b. False
15. At any time, manufacturers may submit updates to their dossiers in anticipation of a therapeutic class review by a health system P\&T Committee. This statement is:
a. True
b. False

16. Section 3.0 should contain all the following types of key clinical studies EXCEPT:

a. Relevant safety, efficacy, effectiveness and comparative effectiveness studies

b. Phase 3 clinical trials

c. Relevant Phase 2 trials

d. Pharmacologic, pharmacokinetic studies, and Phase 1 studies

e. Relevant studies conducted outside the U.S.

17. Regarding summaries of key clinical studies, which of the following statements is TRUE?

a. Manufacturers are to include relevant Cochrane Collaboration systematic reviews

b. Summaries are to include all relevant positive and negative findings

c. Summaries should delineate primary and secondary endpoints

d. Summaries of trial results of key comparator products are desirable but not required

e. All of the above

18. Regarding the modeling report, which of the following statements is FALSE?

a. A societal perspective is recommended for the primary analysis; the payer perspective may be presented as a secondary analysis.

b. Manufacturers should perform sensitivity analyses on all parameters.

c. Cost-effectiveness analyses should depict the primary treatment options and the treatment process for each option.

d. Manufacturers should provide a figure detailing the structure of the model such as a decision tree.

e. Models that have been previously developed may be adapted for use according to the AMCP Format.

19. Manufacturers are to provide reprints of all studies summarized in Sections 3 and 5 and links to the original sources if they are free of charge.
a. True
b. False

20. Which of the following analytic techniques express both net costs and benefits of the health intervention in monetary units?
a. Cost-consequence analysis
b. Cost-effectiveness analysis
c. Cost-minimization analysis
d. Cost-benefit analysis
e. Cost-utility analysis

To complete this continuing education activity, go to www.amcp.org (CE/CME Center) to access the posttest and evaluation form. 


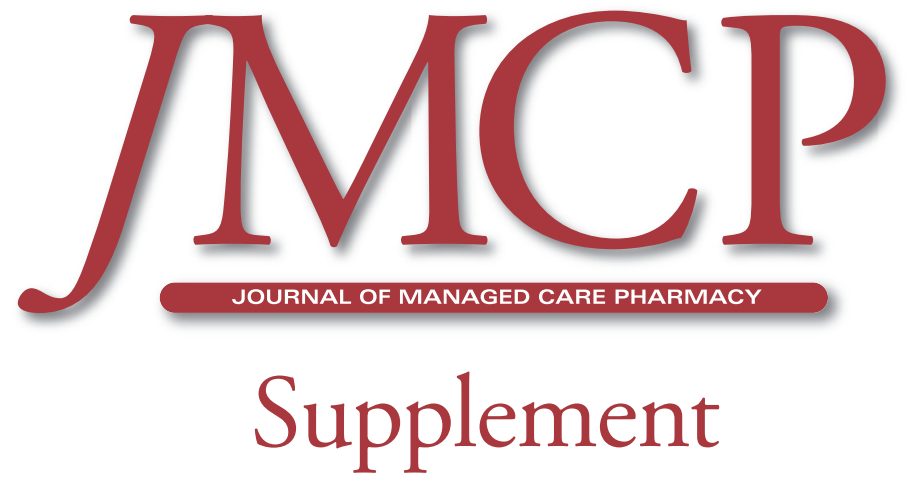

